Confidential Enquiries into Maternal Deaths and Morbidity 2013 15. MBRRACE-UK, 2017.

3 Department of Health. Safer maternity care: progress and next steps. London: DH, 2017.

\section{Collaborative research has direct patient benefit and merits recognition}

We are pleased that Donovan and Sangha noted our call for collaborative research to be fully recognised by postgraduate training selection panels. ${ }^{1}$ However, we are concerned by their conflation of research collaboratives with 'soft target' journals which are financially motivated to publish work of such low scientific value, that it would not pass through peer review into a mainstream journal. ${ }^{2}$

Trainee research collaboratives conduct high impact multicentre studies, such as the West Midlands Research Collaborative's randomised controlled trial 'Dexametasone reduces emesis after major surgery' (DREAMS). This 1350 patient trial demonstrated that administration of dexamethasone at induction reduces postoperative nausea and vomiting by one-third. ${ }^{3}$ In the evidencebased medicine era, only multicentre studies like DREAMS can change clinical practice. By necessity these are delivered by large, complex teams; 300 coinvestigators contributed to DREAMS across 45 hospitals.

Although DREAMS recruited fewer than 5 patients per coinvestigator, opening the trial at each site, completing mandatory training, screening patients for eligibility, consenting and randomising patients, delivering interventions, and completing follow-up required a significant investment of time over many months; this does not equate to the 'minimal effort' outlined in Donovan and Sangha's letter.

Regardless of whether individuals participate 'for the love of it', research collaboratives enable students and trainees to lead and contribute to research that has the potential to improve patient care. Furthermore, it equips them with practical academic skills, ${ }^{4}$ promoting further engagement with research and quality improvement across the NHS. ${ }^{5}$ STARSurg's International Journal of Surgery letter argued that it is in patients' interests for participation in high-quality research such as DREAMS to be fairly recognised by selection bodies on par with other types of publication. ${ }^{6}$ This position is supported by the core surgical training, neurosurgery, urology, and general surgery Specialty Association Committees who now recognise collaborative research in award of Certificates of Completion of Training.

We agree that it would be inappropriate to award points in selection processes for short letters. However, recognition of collaborative research is not only wholly merited, but also essential to ensure trainees continue to contribute to high-impact research for patient benefit.

\section{Conflicts of interest}

DN was the guarantor for Student Audit and Research in Surgery's (STARSurg) International Journal of Surgery letter. JCG is the current senior lead of STARSurg (www.starsurg.org, @STARSurgUK).

DMITRI NEPOGODIEV* Academic Department of Surgery, University of Birmingham, Birmingham, UK
JAMES C GLASBEY*

Academic Department of Surgery, University of Birmingham,

Birmingham, UK

*both authors contributed equally

\section{References}

1 Donovan K, Sangha G. The application game. Clin Med 2017;17:586.

2 Shen C, Björk BC. 'Predatory' open access: a longitudinal study of article volumes and market characteristics. BMC Med 2015;13:230.

3 DREAMS Trial Collaborators and West Midlands Research Collaborative. Dexamethasone versus standard treatment for postoperative nausea and vomiting in gastrointestinal surgery: randomised controlled trial (DREAMS Trial). BMJ 2017;357:j1455.

4 Chapman SJ, Glasbey JC, Khatri C et al. Promoting research and audit at medical school: evaluating the educational impact of participation in a student-led national collaborative study. BMC Med Educ 2015;15:1.

5 Nepogodiev D, Chapman SJ, Kolias AG et al. The effect of trainee research collaboratives in the UK. Lancet Gastroenterol Hepatol 2017:2:247-8.

6 Collaborative STARSurg. Students' participation in collaborative research should be recognised. Int J Surg 2017;39:234-7.

\section{Response}

We welcome Nepogodiev and Glasbey's response to our letter. The central thesis of our original piece was that points-based selection criteria for training posts are flawed because they are founded on misleading metrics and do not allow for adequate assessment of individuals' contribution to research, or of their motivation. While we agree that research collaboratives produce high-quality and high-impact research, we do think that these issues become particularly apparent when considered in the context of a large student or junior doctor research collaborative. This is because, by their very nature, collaboratives rely on distributing a large amount of work over a very large number of individuals, thus reducing each individual's proportional contribution. One could easily envisage a situation where a canny (but entirely reasonable) junior doctor would take advantage of this distribution of labour to score a 'point' which was earned with little work, and the wrong motivation. Such a candidate could easily be identified in a less structured application process with more attention paid to white space and freely flowing interview; however, he or she could not be distinguished by an algorithm based on points-for-publications.

Collaborative research is changing medicine for the better and should be recognised. The problem lies with application systems which only see points and not individuals.

\section{KILLIAN DONOVAN} GINA SANGHA

Oxford University Clinical Academic Graduate School, Oxford, UK

\section{The hazards of neglecting the skin}

Editor - Elder et $a l^{1}$ have addressed the important but overlooked subject of the physical examination. However, as a dermatologist I was disappointed to discover that of 58 different components of the physical examination that they considered, ranging from ophthalmoscopy to digital rectal examination, examination of the largest and most accessible organ of the body, the skin, had been entirely overlooked, other than 'skin turgor'.

Cutaneous manifestations of systemic disease are numerous and common; the fingernails alone may reveal splinter haemorrhages 